\title{
CYCLOSPORIN H: A NOVEL ANTI-INFLAMMATORY THERAPY FOR INFLUENZA FLU PATIENTS
}

\author{
By
}

SALWA A. ELGEBALY ${ }^{1 *}$, TAMER ELBAYOUMI ${ }^{2}$, and DONALD L. KREUTZER ${ }^{3}$

Nour Heart, Inc. ${ }^{1}$, Biotech Company, Germantown, Maryland, United States of America; College of Pharmacy-Glendale/Nanomedicine Center of Excellence in Translational Cancer Research ${ }^{2}$, Midwestern University, Arizona, United States of America; University of Connecticut Faculty of Medicine, Farmington, Connicticut ${ }^{3}$, United States of America ("Correspondance:selgebaly@nourheart.com)

\begin{abstract}
Relatively little is known about the inflammatory mediators and mechanisms that drive the progression of influenza flu infection to cytokine storm, lung dysfunction, organ failure, and ultimately death. Vaccines and antiviral medications cannot control the excessive host inflammatory response associated with severe influenza flu infection.

Studies by Elgebaly et al demonstrated the rapid release of a potent inflammatory mediator, recently named Nourin, by local mammalian tissues in response to injury and infection. Nourin is a formyl peptide that acts through the formyl peptide receptor (FPR) on phagocytic leukocytes. As an initial signal in the innate immunity, Nourin stimulates leukocyte chemotaxis, induces acute and chronic inflammation, and stimulates the release of a number of the cytokine storm mediators from monocytes, neutrophils and endothelial cells. Furthermore, Nourin detected in plasma samples from patients with severe influenza infection was much higher compared to moderate influenza.

The Nourin antagonist, Cyclosporin $\mathrm{H}$, is a potent anti-inflammatory compound, which acts as a specific competitive antagonist of formyl peptides on the formyl peptide receptor (FPR) on phagocytic leukocytes. Cyclosporin $\mathrm{H}$ completely blocked neutrophil chemotaxis induced by: (a) the standard formyl peptide, f-MLF, (b) the Staphylococcus aureus bacteria-derived formyl peptide Phenol-soluble modulins, such as PSM3a, plus(c) the host-derived Nourin released by: (1) cultured epithelial cells infected with the PR8 H1N1 influenza virus for 6 to 24 hours, (2) Nourin detected in the serum of mouse model of H1N1 Swine flu influenza infection for 6 hours , along with (3) Nourin detected in plasma samples collected from severe and moderate influenza patients. Furthermore, in-vivo treatment by Cyclosporin H in the mouse model of H1N1 Swine flu influenza infection for 5 days markedly reduced lung inflammation and endothelial cell damage. Thus, two clinical applications for Nourin and its antagonist Cyclosporin $\mathrm{H}$ are proposed:

Diagnostic Application: The blood Nourin test can be used as a key inflammatory biomarker for "early" detection and monitoring of influenza flu patients proceeding to hyperactive inflammation and, thus, permitting early crucial anti-inflammatory therapy.

Therapeutic Application: Cyclosporin $\mathrm{H}$ will specifically block Nourin as an important initial stimulant of cytokine mediators, and thus can control the development and progression of cytokine storm plus organ inflammation, which usually initiates 3 to 8 days post influenza. Since Cyclosporin $\mathrm{H}$ does not target the virus, it will not develop drug resistance and will reduce the host uncontrolled inflammatory response, induced by both new strains of flu viruses and existing viruses with mutations.

Keywords: Influenza Flu virus, Cytokine Storm, Formyl Peptide Nourin, Formyl Peptide Receptor, Formyl Peptide Competitive Antagonist, Cyclosporin H, Anti-Inflammatory Therapy, Diagnostic Inflammatory Biomarker, Methicillin-resistant Staph. aureus (MRSA).
\end{abstract}

\section{Introduction}

A zoonotic disease is a disease that can be passed between animals and humans. Zoonotic diseases can be caused by viruses, bacteria, parasites, and fungi. These diseases are very common. Scientists estimate that more than 6 out of every 10 infectious diseases in humans are spread from animals. 
Avian influenza (AI) is an infectious viral disease of birds (especially wild water fowl such as ducks and geese) which has also been reported to cross the species barrier and cause disease in humans. The highly pathogenic avian influenza flu virus A (H5N1) is still a potential threat to public health worldwide with pandemic potential, because it continues to circulate widely in some poultry populations, most humans likely have no immunity to them, and they can cause severe disease and death in humans. While vaccines and antiviral drugs are currently used to treat influenza flu patients, anti-inflammatory therapy could offer a new strategy to treat the host-overactive inflammation associated with influenza flu virus infection.

The clinical manifestations associated with $\mathrm{H} 1 \mathrm{~N} 1$ infection in humans range from asymptomatic infection to mild upper respiratory illness, severe pneumonia, and multiple organ failure. Influenza A virus infection is still a major cause of respiratory tract infection in Egyptian children (Adel Khattab et al, 2013). It should be considered in all cases with cough and febrile episodes, and influenza-like symptoms, even post Swine flu pandemic.

Background: The Nourin family represents a group of $3 \mathrm{KDa}$ formyl peptides released by local tissues in response to diverse types of injury (Elgebaly et al, 2016 Review, Elgebaly et al, 1987; 1989; Kozol et al, 1989; Ruby et al, 1990; Elgebaly et al, 1991; 1994; 2002; Christenson et al, 2004; Elgebaly et al, 2010; Elgebaly et al, 2012). In earlier publications, Nourin was referred to as "tissuederived neutrophil chemotactic factor".

Nourin is released in response to injury such as ischemia, chemical agents (hydrogen peroxide, sodium hydroxide, and citric acid), physical trauma (scraping), ultraviolet exposure, vitamin A deficiency with minor physical injury, shear stress pressure, infectious agents, including bacterial endotoxin and influenza flu virus (Elgebaly et al, 2010; Elgebaly et al, 2012).
Although Nourin released by various tissues shared the $3 \mathrm{KDa}$ molecular weight with the common motif of formyl-methionyl at N-terminus that acts through the formyl peptide receptor (FPR) on leukocytes yet, they differ in their isoelectric points, organic solubility, heat sensitivity, and mode of release (Elgebaly, 1997; 2010; Elgebaly et al, 2010). To date, the many tissues studied that release Nourin in response to injury include: heart, coronary arteries, vein grafts, cornea, conjunctiva, retina, stomach, urinary bladder, brain and spinal cord, as well as cultured corneal endothelial cells, kidney epithelial cells, and human bladder fibroblasts (Elgebaly et al, 2010; 2012).

In-vitro, Nourin stimulates leukocyte chemotaxis and the release of a number of cytokine and chemokine mediators as well as digestive enzymes and free radicals by human monocytes, neutrophils and vascular endothelial cells (Elgebaly et al, 1989; 1992; 1993; Tyles et al, 1994; 1995). These included: interleukin-1 (IL-1 $\beta$ ), interleukin-8 (IL8 ), tumor necrosis factor (TNF- $\alpha$ ), adhesion molecules by neutrophils (LECAM) and human aortic endothelial cells (ICAM-1and ELAM-1), collagenase type IV, N-acetyl-Bglucosaminidase, gelatinases and superoxide anion.

In-vivo, the injection of tissue-derived Nourin induced acute and chronic inflammatory responses. For example: (a) cardiacderived Nourin injected into rabbit skin resulted in acute inflammation within 30 minutes to 24 hours (Elgebaly et al, 1992), (b) corneal-derived Nourin injected into rabbit corneas resulted in acute inflammation with 2 hours characterized by neutrophil infiltration and edema (Allam et al, 1990), and (c) conjunctival-derived Nourin injected into rabbit conjunctiva resulted in acute and chronic inflammation characterized by leukocyte infiltration and deposition of fibrin and collagen (Ehlers et al, 1990; 1991).

Since Nourin is rapidly released by injured tissue and stimulates leukocyte chemotaxis and the secretion of a number of cyto- 
kine and chemokine mediators as well as digestive enzymes and free radicals characteristic of cytokine storms, it is believed that Nourin plays an important role as an "initial signal" in the cascade of events that lead to the initiation and amplification of leukocyte recruitment and activation leading to severe inflammation and tissue damage. Furthermore, since Nourin contributes to the induction of tissue acute and chronic inflammatory responses, it can be characterized as an Alarmin and used as a crucial therapeutic target.

Cyclosporin H (Cook, 1983; WenzelSeifert, 1991; Bavandi et al, 1992; WenzelSeifert et al, 1993; De Paulis et al, 1996) is a member of competitive antagonists of the formyl peptide receptor (FPR) on leukocytes. It was reported that Cyclosporin $\mathrm{H}$ is a potent anti-inflammatory agent since it significantly inhibits FMLP-induced neutrophil and basophil chemotaxis and superoxide anion (O2-) formation. Unlike Cyclosporin, A which is an immunosuppressant, Cyclosporin $\mathrm{H}$ is not recognized as an immunosuppressor (Cook, 1983; WenzelSeifert, 1991; Bavandi et al, 1992; WenzelSeifert et al, 1993; De Paulis et al, 1996). Specifically, Cook RM, 1983 demonstrated that oral administration of the immunosuppressive fungal metabolite Cyclosporin A increased the mortality of Balb/c mice infected intra-nasally with influenza $\mathrm{A} / \mathrm{Hong}$ Kong/1/68 (H3N2) virus. Cyclosporin A also increased the amount of virus that could be recovered from the lungs of infected mice, and delayed the rate at which it was eliminated (Cook, 1983).

The present study demonstrated that Cyclosporin $\mathrm{H}$ and other members of competitive antagonists of the formyl peptide receptor (FPR) on leukocytes including t-BocFLFLF, Spinorphin and the soluble FPR fragment 17 aa loop peptide have markedly inhibited Nourin-induced leukocyte chemotaxis in-vitro (Elgebaly et al, 2010; 2012). The study also demonstrated that the Nourin release by injured tissues is not influenced by the treatment of anti-inflammatory drugs such as steroids and NSAIDS (Ibuprofen) (Elgebaly et al, 1988; 1994; Tyles et al, 1994). Alcohol treatment, on the other hand, reduced the levels of Nourin by ischemically-injured gastric tissue (Kozol et al, 1989). Interestingly, the treatment by Cyclocreatine and Cyclocreatine phosphate preserved high levels of Adenosine Triphosphate (ATP) in ischemic heart tissue, reduced the formation of Nourin by ischemic myocardium and markedly reduced post-ischemic cardiac inflammation. In fact, myocardial perseveration of during ischemia by Cyclocreatine and Cyclocreatine phosphate resulted in significant restoration of cardiac function during reperfusion (Elgebaly et al, 2016).

Hypothesis: The influenza virus infection of airway epithelial cells will trigger cell injury and the release of proinflammatory mediators including the formyl peptide Nourin and that the Nourin antagonist Cyclosporin $\mathrm{H}$ will inhibit Nourin's chemotactic activity in-vitro and reduce lung inflammation in-vivo.

Objectives: 1- To determine the in-vitro release of the formyl peptide Nourin in supernatant solutions of cultured epithelial cells (MDCK) infected with the H1N1 influenza virus (PR8) for 1 to $24 \mathrm{hrs}$. 2- To determine the in-vivo release of the formyl peptide Nourin in serum samples of mice infected with the H1N1 influenza virus for only 6 hours. 3- To establish the detection of the formyl peptide Nourin in plasma samples of patients with severe and moderate influenza flu infection and whether there is a differential Nourin levels between severe and moderate influenza patients infected with the H1N1 influenza virus. 4- To determine whether the formyl peptide antagonist, Cyclosporin $\mathrm{H}$, inhibits in-vitro leukocyte chemotactic activity stimulated by Nourin in response to influenza flu infection in cultured epithelial cells (MDCK), mice and patients. 5- To determine whether the formyl peptide competitive antagonist, Cyclosporin $\mathrm{H}$, inhibits lung inflammation in-vivo in the mouse model of H1N1 Swine flu influenza infection for 5 days. 6- To determine whether 
the formyl peptide antagonist Cyclosporin $\mathrm{H}$ inhibits in-vitro leukocyte chemotactic activity stimulated by the potent formyl peptide chemotactic factor Phenol-soluble modulins, PSM3a, released by the $S$. aureus bacteria.

Methodology: 1- Cultured Epithelial Cell Studies: Cultured epithelial MDCK cells were infected with H1N1 (PR8) influenza virus for $1,3,6,12$, \& 24 hrs. Each $\mathrm{H}_{1} \mathrm{~N}_{1}$ infected cell supernatant was assayed both in the presence and absence of the formyl peptide specific antagonist Cyclosporin $\mathrm{H}\left(5 \times 10^{-}\right.$ $\left.{ }^{6} \mathrm{M}\right)$. Supernatant solutions were evaluated for chemotactic activity both undiluted (neat) and diluted 1/10 in hanks balance salt solution (HBSS). Samples were assayed for chemotactic activity using standard Neuroprobe chemotaxis system (Gaithersburg, Maryland) and human leukocytes as indicator cells.

2- Mice In-vivo Studies: Balb/C female, 5 weeks old mice $(n=4)$ were anesthetized with isofluorane and intranasaly inoculated with 10 MLD50 of WSN (mouse adapted A/ WSN/33 strain, $\mathrm{H}_{1} \mathrm{~N}_{1}$ ) in 50ul PBS (designated WSN-1 thru WSN-4). Four mice received sham treatment (control group) without $\mathrm{H}_{1} \mathrm{~N}_{1}$ virus (designated Mock-1 thru Mock-4). Six hours post virus or sham treatment, blood samples were collected from mice and the serum was stored at $-70^{\circ} \mathrm{C}$ until used for chemotaxis assay to determine the level of Nourin chemotactic activity and the ability of its antagonist Cyclosporin $\mathrm{H}\left(5 \times 10^{-6} \mathrm{M}\right)$ to inhibit that activity. Serum samples were diluted in HBSS at a dilution of 1/7.

3- Influenza Patients' Studies: This preliminary study was conducted on severe H1N1 influenza flu patients admitted to the ICU with encephalopathy or respiratory failure as well as moderate influenza patients and patients with RSV who were admitted to the hospital with fever or wheezing. The plasma samples were obtained from five patients with severe H1N1 influenza flu and three moderate patients with H1N1 influenza flu, as well as from two patients with respiratory syncytial virus (RSV) infection. The presence and level of Nourin-stimulated chemotactic activity in plasma samples were measured after dilution 1/9 in HBSS in the presence and absence of the Nourin inhibitor Cyclosporin $\mathrm{H}$ at $10^{-5} \mathrm{M}$.

4- Mice Cyclosporin H Anti-inflammatory Studies: To specifically test the ability of Cyclosporin $\mathrm{H}$ to inhibit lung inflammation in-vivo in the H1N1 Swine influence flu mouse model mice $(n=5)$ were treated with Cyclosporin $\mathrm{H}$ at $700 \mathrm{ug} / \mathrm{mouse} /$ day $(35 \mathrm{mg} /$ $\mathrm{kg}$ in mouse) which is equivalent to the low dose of $2.8 \mathrm{mg} / \mathrm{kg} /$ day for human. Cyclosporin $\mathrm{H}$ was administered via intraperitoneal injections (IP) twice daily every 12 hours for one day prior to influenza inoculation and then twice daily for the next 5 days. Control mice $(n=5)$ received saline and it was admistered via intraperitoneal injections (IP) twice daily every 12 hours for one day prior to influenza inoculation and then twice daily for the next 5 days. The Cyclosporin $\mathrm{H}$ treated mice and control saline-treated mice were sacrificed at day 5 and lungs were removed and processed for histological analysis (Elgebaly et al, 2010; 2012).

5- Methicillin-resistant $S$. aureus (MRSA) limited study caused sepsis in hospitals and communities (CA-MRSA). Phenol-soluble modulins such as PSM3a are formyl peptides secreted by $S$. aureus bacteria as key Methicillin-resistant Staph. aureus (MRSA) infections cause sepsis in hospitals and communities (CA-MRSA) and they have potent leukocyte chemotactic activity for the phagocytic leukocyte. Since S. aureus infection also results in over-active host inflammatory response (cytokine storms), formation of tiny blood clots leading to tissue ischemia, organ failure and death, the study tested whether Cyclosporin $\mathrm{H}\left(10^{-4} \mathrm{M}\right.$ $\left.\& 10^{-5} \mathrm{M}\right)$ as a formyl peptide competitive antagonist on leukocyte FPR would inhibit PSM3a-induced chemotactic activity invitro.

\section{Results and Discussion}

The study showed that treating human leukocytes with Cyclosporin $\mathrm{H}$ at $10^{-4} \mathrm{M} \& 10^{-5}$ $\mathrm{M}$ as a formyl peptide competitive antago- 
nist on leukocyte FPR significantly inhibitedfMLP-induced chemotactic activity invitro (Fig. 1). Cultured Epithelial Cell Studies: Nourin was released by cultured canine epithelial MDCK cells in response to infection with the influenza H1N1 (PR8) virus for 1 hour (2.5-fold control value) and continued for an additional 24 hours. Nourin chemotactic activity was inhibited back to control value by Cyclosporin $\mathrm{H}\left(5 \times 10^{-6} \mathrm{M}\right)$ treatment in-vitro (Elgebaly et al, 2010; 2012).

Mice in-vivo studies: higher levels of Nourin (Fig. 2) were detected in serum samples collected from mice infected with the Swine flu H1N1 virus for 6 hours compared to the control group (Elgebaly et al, 2010; 2012). Cyclosporin $\mathrm{H}\left(5 \times 10^{-6} \mathrm{M}\right)$ treatment of the mouse serum samples significantly inhibited Nourin chemotactic activity, all the way back to baseline values.

Influenza patients' studies - high level of Nourin was detected in plasma samples collected from patients with severe and moderate influenza infection and to a lesser extent in patients with respiratory syncytial virus (RSV) infection. The Nourin level in severe influenza patients was double the level in plasma samples collected from the moderate patients (Figure 3). Furthermore, the Nourin competitive antagonist, Cyclosporin $\mathrm{H}$, at $10^{-5} \mathrm{M}$ significantly inhibited neutrophil chemotaxis induced by host-derived Nourin, as detected in all influenza patients' samples.

Mice Cyclosporin $\mathrm{H}$ anti-inflammatory studies, demonstrated that treating mice infected with the Swine flu H1N1 virus with Cyclosporin $\mathrm{H}$ for 5 days significantly reduced lung inflammation as described in Figure 4. There was less leukocyte infiltration into the lung tissues of the Cyclosporin $\mathrm{H}$-treated lungs compared to the H1N1 influenza saline-treated mice, evident through reduced perivascular lymphocytic infiltrates, reduced perivascular neutrophil infiltrate and pavementing and reduced endothelial cell damage.
Unlike Cyclosporin A, which was reported to increase the amount of influenza virus that could be recovered from the lungs of infected mice (Cook, 1983), the present result showed that Cyclosporin $\mathrm{H}$ did not change the viral load in the infected mice at day 5, when compared to control group, supporting that Cyclosporin $\mathrm{H}$ is not an immunosuppressor. Accordingly, this preliminary study suggests that: a- Cyclosporin $\mathrm{H}$ does not target the influenza virus, since it did not reduce the viral titer; meanwhile there is a reduction in inflammation and necrosis, b- Cyclosporin $\mathrm{H}$ reduced inflammation by blocking the action of the formyl inflammatory mediator "Nourin", which was early released by 6 hours in response to viral infection, c- Cyclosporin $\mathrm{H}$ is not an immunosuppressor, because it did not increase viral replication when it was administered one day before influenza viral inoculation and continued for an additional 5 days.

Methicillin-resistant $S$. aureus (MRSA) limited study demonstrated that Cyclosporin $\mathrm{H}$ at concentration of $10^{-4} \mathrm{M}$ inhibited leukocyte chemotactic activity induced by PSM3a by more than $90 \%$ and by $50 \%$. at a concentration of $10^{-5} \mathrm{M}$ (Fig. 5).

\section{Conclusion}

The outcome results proved the rapid and sustained release of the formyl peptide inflammatory mediator Nourin both in-vitro using cultured epithelial cells, and in-vivo in mice after H1N1 influenza flu infection. Furthermore, higher levels of Nourin were detected in the influenza patients' plasma samples from severe influenza compared to patients with moderate influenza flu. Cyclosporin $\mathrm{H}$ inhibits leukocyte chemotactic activity induced in-vitro by Nourin detected in cultured epithelial cell supernatant solutions and mouse sera after H1N1 influenza flu infection, and plasma samples from patients with severe and moderate H1N1 influenza infection. Cyclosporin $\mathrm{H}$ also markedly inhibited leukocyte chemotactic activity induced by $S$. aureus formyl peptide product PSM3a. 
In-vivo, daily treatment of Cyclosporin $\mathrm{H}$ for 5 days inhibited lung inflammation in mice infected with the Swine H1N1 influenza flu virus. Cyclosporin $\mathrm{H}$ did not target the influenza virus and did not reduce the mice immunity, but rather specifically blocked the activity of the inflammatory Nourin, resulting in less lung inflammation.

It is still not clear what are the endogenous inflammatory mediators that modulate the initial inflammatory responses (innate immunity) during Swine H1N1 influenza flu infection and Staph. aureus infection and forces the immune system into overload causing organ failures and in some cases death. Even less is known about the early immune cascade of events that tilt the balance to either immune protection with resolution and recovery or to the devastating overactive inflammatory response led to immunopathology disease and death(Fig. 6).

Since Nourin is rapidly released by local tissues in response to injury and infections, plus it stimulates the release of high levels of cytokine storm mediators (IL-8, IL- $1 \beta$, TNF- $\alpha$, adhesion molecules, free radicals, and digestive enzymes) by neutrophils, monocytes, and vascular endothelial cells, it might likely "amplify" the host inflammatory response leading to immunopathology and diseases, which are characterized by cytokines dysfunction, organ failure, and ultimately death (Fig. 6). Nourin can, therefore, be considered an endogenous therapeutic target.

Future diagnostic and therapeutic applications are as follows: A- The blood Nourin test can be used as a key inflammatory biomarker for early detection and monitoring of influenza flu patients proceeding to hyperactive inflammation and, thus, permitting early crucial anti-inflammatory therapy. B - Cyclosporin $\mathrm{H}$ will have the advantage of blocking Nourin as an "early" key mediator and control the "development" and "progression" of inflammation and cytokine storm initiated 3-8 days post influenza (Fig. 7). Additional advantages of Cyclosporin $\mathrm{H}$ include: 1- Unlike the concern of not making enough vaccines to protect significant numbers of people, Cyclosporin $\mathrm{H}$ is a small molecule and can be easily prepared in large scale for worldwide consumption, at a low cost. 2- Unlike antiviral drugs, such as Tamiflu, which target the virus, Cyclosporin $\mathrm{H}$ did not change the viral load indicating that Cyclosporin $\mathrm{H}$ will not develop drug resistance and it will continue to reduce the host uncontrolled inflammatory response induced by new strains of flu viruses and existing viruses with mutations. 3- Unlike Tamiflu, which must be taken within one to two days after the appearance of symptoms, Cyclosporin $\mathrm{H}$ does not have this strict time limitation and it can be administered both shortly after symptoms, as well as days after infection to combat cytokine storms initiated between days $3 \& 8$ post influenza. 4- Unlike current cortisone treatments, which can cause immunosuppression and subject patients to additional infections and cancer, Cyclosporin $\mathrm{H}$ will specifically inhibit the tissue-derived inflammatory mediator "Nourin", which induces tissue inflammation and cytokine storms without affecting the host defense immune system.

\section{References}

Bavandi, A, et al, 1992: Diet-induced dermatitis response of hairless rats to systemic treatment with cyclosporin A (Sandimmun), Cyclosporin H and FK506, Exp Dermatol, 1:199-205.

Cook RM, 1983: Activity of cyclosporin A in experimental influenza virus infection in mice. Agents Actions 13, 1:98-100.

De Paulis, A, Ciccarelli, A, de Crescenzo, G, Cirillo, R, Patella, V, et al, 1996: Cyclosporin $\mathrm{H}$ is a potent and selective competitive antagonist of human basophil activation by $\mathrm{N}$ formyl-methionyl-leucyl-phenylalanine. J. Allergy Clin. Immunol. 98, 1:152-64.

Elgebaly, SA, Poston, R, Elbayoumi, T, Kreutzer, D, 2016: Nourexal ${ }^{\mathrm{M}}$ (Cyclocreatine Phosphate): A new cardioprotective therapy against ischemia/reperfusion injury. Journal Global Cardiology Science and Practice. Review Article

Elgebaly, SA, Herkert, N, O'Rourke, J, Kreutzer, DL, 1987: Characterization of neutrophil and monocyte-specific chemotactic factors de- 
rived from isolated corneas in response to immunologic injury. Am. J. Pathol. 126:22-32.

Elgebaly, SA, Masetti, P, Forouhar, F, Rossomando EF, Cohen A, 1988: Ibuprofen fails to inhibit the release of the tissue-derived neutrophil chemotactic factors "Nourin" from ischemic hearts. XXVI World Cong. Int. Coll. Surg. July 3-9, Milan, Italy.

Elgebaly, SA, Masetti, P, Allam M, Forouhar F, 1989: Cardiac-derived neutrophil chemotactic factors: preliminary biochemical characterization. J. Mol. Cell Cardiol. 21:585-93.

Elgebaly, SA, Cohen, A, Allam, M, Yekta, N, 1989: Cardiac derived inflammatory mediators. J. Mol. Cell Cardiol. 21, 11:S171.

Elgebaly, SA, Miano, D, Fishman, J, Kreutzer, DL, 1990: Cornea-derived neutrophil chemotactic factors: Intracellular synthesis and release. Curr. Eye Res. 9, 9:839-45.

Elgebaly, SA, Allam, ME, Walzak, MP, Oselinksy, D, 1991: Urinary neutrophil chemotactic factors in interstitial cystitis patients and a rabbit model of bladder inflammation. J. Urol. 147:206-11.

Elgebaly, SA, Hashmi, F, Houser, S, Allam, ME, 1992: Cardiac-derived neutrophil chemotactic factors: detection in coronary sinus effluents of patients undergoing myocardial revascularization. J. Thorac. Cardiovasc. Surg. 103, 5: 952-9.

Elgebaly, SA, House, SL, Tyles, E, El-Kerm, AF, Mauri, F, et al, 1992: Characterization of a novel small molecular weight neutrophil chemotactic factor human hearts. Circulation 86, 4:

Elgebaly, SA, Tyles, E, Houser, SL, El-Kerm, AF, Mauri F, 1993: Partial purification of a novel cardiac-derived neutrophil chemotactic factor: Nourin-1. Circulation 88, 4:

Elgebaly, SA, Houser, SL, El-Kerm, AF, Doyle, K, Gillies, C, et al, 1994: Evidence of cardiac inflammation after open heart surgery. Ann. Thorac. Surg. 57:35-41.

Elgebaly, SA, Miano, D, Ehler, W, Rahhal F, El-Kerm A, 1994: The induction of anterior chamber inflammation by factors released from hydrogen peroxide-injured corneas: effect of dexamethasone and indomethacin. Ocul. Pharmacol. 10, 1:295-305.

Elgebaly, SA, 2010: Diagnostic Marker (Patent \#7,659,091).

Elgebaly, SA, Kreutzer, DL, 2002: Release of a potent inflammatory mediator from ischemic brain. $27^{\text {th }}$ Int. Stroke Conf. American heart Association, Austin, Texas, February.

Christenson, R, Elgebaly, SA, 2004: Nourin-1: The "Holy Grail" for Assessing Myocardial Ischemia. Advance 16, 1:19-21.

Elgebaly, SA, Schiffmann, E, 2010: Mitochondrial Marker of Ischemia (Patent \#7,662,571).

Elgebaly, SA, Perez, D, Sullivan, K, Whitaker, C, Caspe, S, et al, 2010: Nourexin-4: A novel anti-inflammatory therapy for influenza flu: Presented to the American Association of Immunologists Meeting in Baltimore, Maryland. Elgebaly, SA, Schiffmann, E, 2012: Methods to Prevent \& Treat Diseases (Patent \# 8,288, 350).

Ehlers, W, Donshik, PC, Gillies, C, Forouhar, F, Allam, ME, et al, 1990: The induction of an inflammatory reaction (Similar to Giant Papillary Conjunctivitis) by chemotactic factors derived from conjunctival cells. Investigative Ophthalmology and Visual Science (abstract), 31, 4: 241.

Ehlers, W, Williams, W, Elgebaly, SA, 1991: Conjunctiva-derived neutrophil chemotactic factors: preliminary biochemical characterization. CLAO J 17:658.

Kozol, RA, Downes, RA, Kreutzer, DL, Wentzel, S, Rossomando, FF, et al, 1989: Release of neutrophil chemotactic factors from gastric tissue: initial biochemical characterization. Dig. Dis. Sci. 34:681-7.

Kozol, RA, Elgebaly, SA, 1989: Ethanol and its effect on mucosal immunity. In: Drugs of Abuse and Immune Function (Watson, R, ed). CRC Press: Colorado.

Ruby, ST, Allam, M, Gallo, M, Barth, S, Elgebaly SA, 1990: Release of chemotactic factors by veins during preparation for arterial bypass. Arch. Surg. 125, 4:481-5.

Osterholm, M, 2005: Preparing for the next pandemic. N. Engl. J. Med.352, 18:1839-43.

Tyles, E, Houser, SL, Shams, N, Consoli, K, Elgebaly, SA, 1994: Coronary sinus effluents from cardiopulmonary bypass patients stimulate the secretion of cytokines and adhesion molecules by vascular endothelial cells. Circulation 90, 4:465.

Tyles, E, Houser, SL, Shams, NK, Consoli, K A, Elgebaly, SA, 1995: Nourin-1 stimulates the secretion of cytokines by neutrophils: Inhibition by Anti-Nourin-1 Antibody. Circulation 92, 8:2. Wenzel-Seifert, K, 1991: Differential inhibition 
of human neutrophil activation by cyclosporins $\mathrm{A}, \mathrm{D}$, and $\mathrm{H}$. Cyclosporin $\mathrm{H}$ is a potent and effective inhibitor of formyl peptide-induced superoxide formation. J. Immunol.147, 6:1940-6

Wenzel-Seifert, K, Seifert, R, 1993: Cyclosporin $\mathrm{H}$ is a potent and selective formyl peptide receptor antagonist: Comparison with N-t-butoxycarbonyl-L-phenylalanyl-L-leucyl-L phenylalanyl-L- leucyl-L-phenylalanine and cyclosporins A. J. Immunol. 150, 10:4591-9.

\section{Explanation of Figures}

Fig. 1: Cyclosporin $\mathrm{H}\left(10^{-4} \mathrm{M}\right.$ and $\left.10^{-5} \mathrm{M}\right)$ inhibits chemotactic activity of human leukocytes induced by chemotactic factor fMLP.

Fig. 2: Cyclosporin $\mathrm{H}\left(5 \times 10^{-6} \mathrm{M}\right)$ inhibits chemotactic activity in mouse sera from mice 6 hours after inoculation with Swine H1N1 influenza flu virus.

Fig.3: Cyclosporin $\mathrm{H}\left(10^{-5} \mathrm{M}\right)$ inhibits leukocyte chemotactic activity induced by Nourin detected in plasmas from influenza patients with severe, moderated and RSV infection.

Fig. 4: Lungs of saline and Cyclosporin $\mathrm{H}$ treated mice infected with Swine H1N1 influenza flu virus.

Fig. 5: Cyclosporin $\mathrm{H}\left(10^{-4} \mathrm{M} \& 10^{-5} \mathrm{M}\right)$ inhibits leukocyte chemotactic activity by PSM3a released by S. aureus bacteria

Fig. 6: Immunopathology during severe viral and bacterial infection.

Fig. 7: Proposed mechanism of cytokine storm evoked by influenza virus and role of Cyclosporin H \& current therapies, modified (Osterholm, 2005).
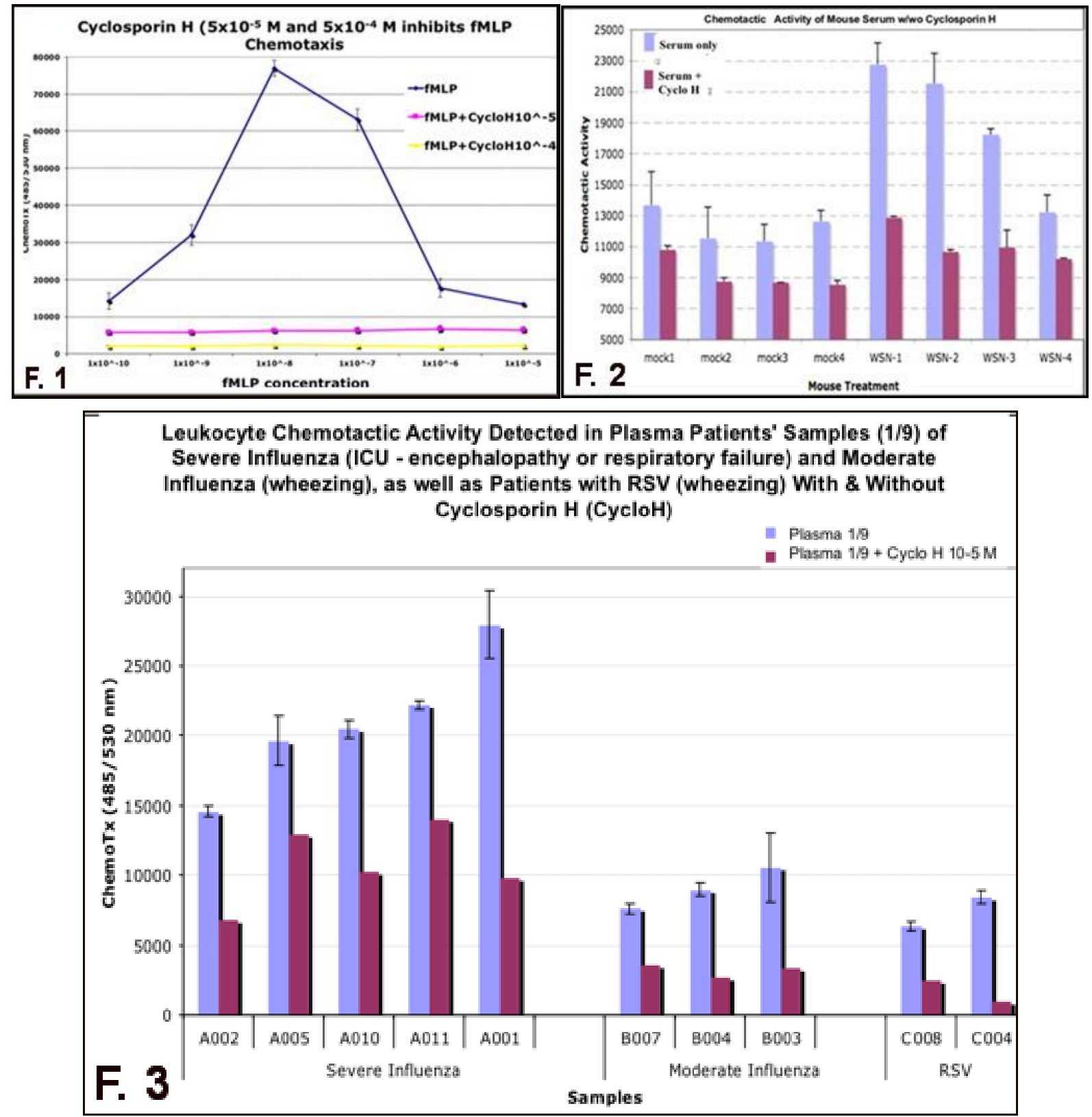

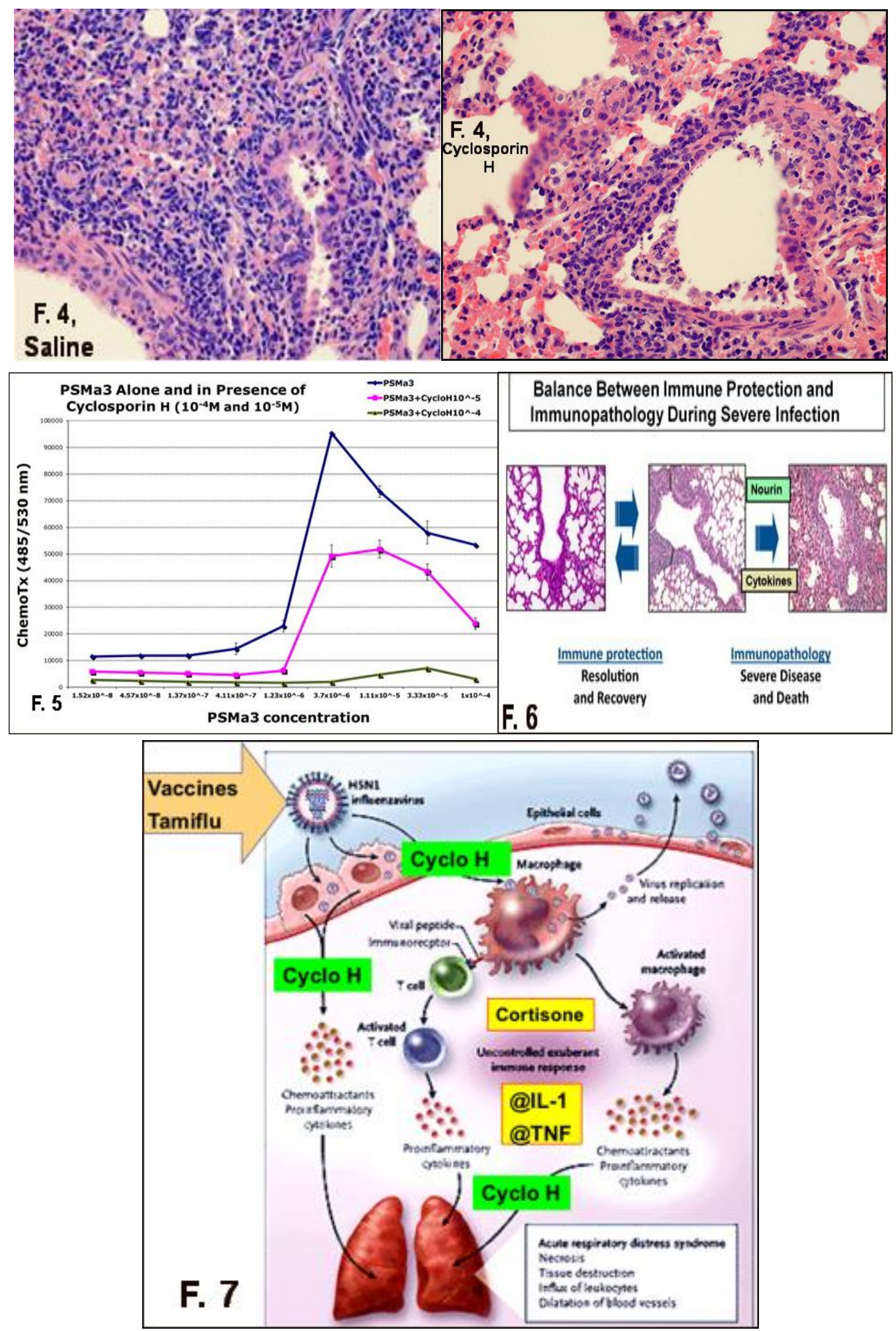